\title{
Anaesthetic management of a patient with critical respiratory compromise resulting from procedure-induced broncho-oesophageal fistulation
}

\section{ABSTRACT}

A 75-year-old woman sustained an unrecognised iatrogenic broncho-oesophageal fistula after repeated oesophageal dilatation. She presented with rapid onset of respiratory distress, requiring intubation and positive pressure ventilation. In the context of massive leakage of tidal volume, a tracheo-oesophageal fistula (TOF) was suspected and confirmed soon after intubation. Due to the site of the fistula (broncho-oesophageal), airway soiling was a concern and adequate ventilation was difficult, necessitating emergency oesophageal stenting.

The principles of peri-anaesthetic management for patients with acquired TOF are to minimise any further airway soiling: antacids, head up position, attempt to position endotracheal tube (ETT) distal to the fistula site or use of a double lumen ETT. One must also be observant for a pneumothorax, which may occur during positive pressure ventilation, prior to stenting or occlusion of the fistula.

\section{INTRODUCTION}

Acquired tracheo or broncho-oesophageal fistulae are potentially life-threating complications of trauma or more commonly malignancy. The most common traumatic cause is endotracheal cuff related necrosis, so anaesthetists should have an awareness of presentation and management of this pathology. Due to persistent airway soiling with gastric contents patients may present with recurrent respiratory tract infections, chest pain and haemoptysis. In ventilated patients a TOF may present with weight loss, increased secretions and repeated pneumonias. Positive pressure ventilation may cause gastric dilatation and diaphragmatic splinting and an audible leak may be heard as gas is expelled via the oesophagus.

Diagnosis is either via bronchoscopy, oeosphagoscopy, Barium swallow or CT. Endoscopic techniques can be used to both identify the site and size of the fistula and effect closure. Fistulae may be treated with surgical correction or stenting of the airway or oesophagus, or parallel stenting of both.

\section{CASE REPORT}

A 75-Year-old marathon runner presented with near total dysphagia developing over recent weeks. She had a background of oesophageal cancer, which had been diagnosed and treated with chemoradiotherapy 2 years prior.

During her inpatient stay, she underwent several oesophogoscopies. The first revealed a narrowing at the previous tumour site and biopsies were taken. Unfortunately her symptoms persisted and the following day she underwent endoscopic balloon dilatation of the oesophageal narrowing using an 'ultra-slim' scope. However, again her symptoms were unresolved and a third endoscopy was performed at midday 3 days after the $1^{\text {st }} O$ G. A re-dilatation was performed for what was described as a 'pin hole' stenosis. At this time a distal mucus tear and bleeding at its site were noted. A routine post procedure chest radiograph (CXR) was unremarkable. 
Four hours later the patient complained of chest pain and difficulty breathing. On the arrival of the medical emergency team she was in marked respiratory distress, gasping, cyanosed and mottled. Oxygen saturations were unrecordable and capillary refill time exceeded 4 seconds despite a blood pressure reading of $213 / 113$. Chest auscultation revealed coarse crepitations globally, worse on the right. No surgical emphysema was observed. An ECG revealed a sinus tachycardia with no ischaemic changes and an arterial blood gas performed on $15 \mathrm{~L}$ oxygen showed profound hypoxia with a respiratory acidosis ( $\mathrm{pH} 7.03, \mathrm{PaO} 26.7, \mathrm{PCO} 213.8, \mathrm{BE}-6.5, \mathrm{HCO} 3$ 19.1). A mobile CXR demonstrated no pneumothorax, pneumomediastinum or pulmonary oedema.

The patient was rapidly stabilised before transfer to the intensive care unit (ICU) where she was intubated (Grade 1 view) with a $7.0 \mathrm{~mm}$ cuffed oral ETT. However a significant leak was immediately audible and tidal volumes inadequate. ETT cuff pressure was confirmed to be within normal limits and the position of the ETT re-confirmed under direct vision. As the audible leak continued the ETT was upsized to $8.0 \mathrm{~mm}$ without appreciable effect. The ETT was then advanced to $24 \mathrm{~cm}$ to produce a purposeful endobronchial intubation because a TOF had been identified as a possible cause at this point.

The oesophageal lumen was assessed using a fibre optic bronchoscope on ICU. Bubbles were observed coinciding with the respiratory cycle, suggestive of a TOF. The gastroenterology consultant was contacted and the patient urgently transferred to theatre for further assessment and management. This fourth OGD confirmed a fistula distal to the narrowing and a $10 \mathrm{~cm}$ oesophageal Nitinol stent was deployed.

Back on the ICU, a left-sided pneumothorax was detected the following day, requiring an intercostal drain. Thereafter oxygen and ventilatory requirements were minimal and she was extubated two days after oesophageal stenting. Due to poor cough and increased effort of breathing, she was reintubated on day 3 before being successfully extubated onto CPAP on day 6 and discharged from ICU at day 10. Dysphagia and odynophagia persisted.

Unfortunately, the biopsy taken at the first OGD demonstrated recurrence of squamous cell carcinoma at the site of narrowing.

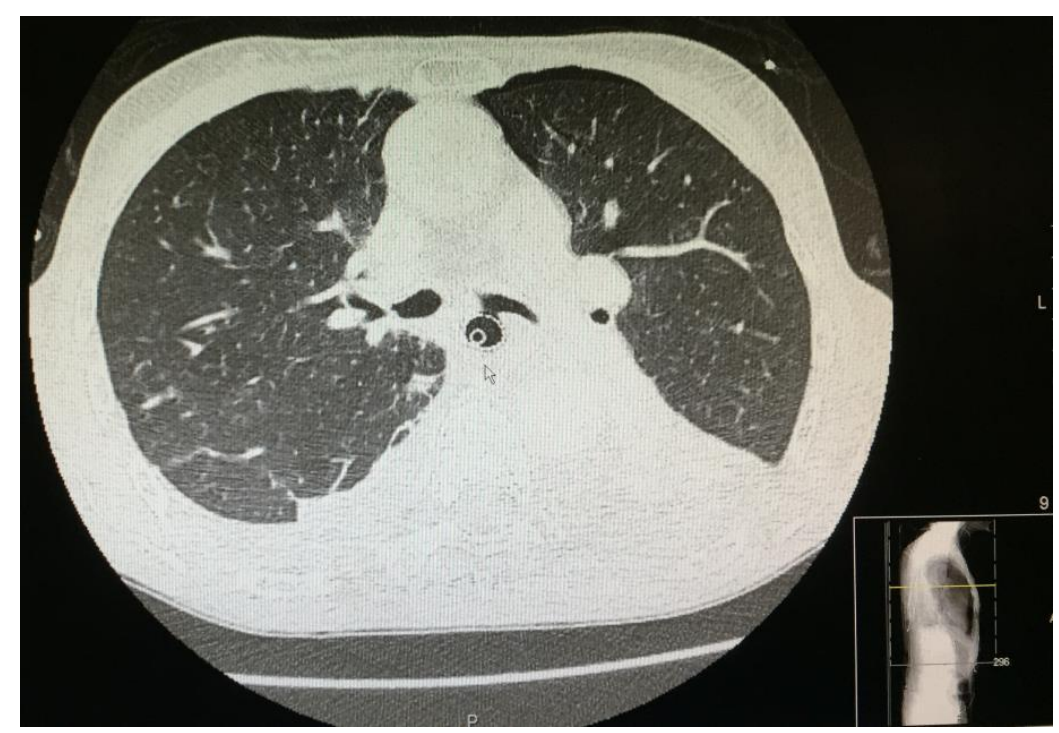

Figure 1. Chest CT demonstrating connection between oesophagus and left main bronchus. The oesophageal stent (and NGT) can be seen within the oesophageal lumen 


\section{DISCUSSION}

Acquired tracheo-oesophageal (TOF) and broncho-oesophageal fistulae (BOF) most commonly occur as a spontaneous complication of local malignancy or result from excessive or prolonged endotracheal cuff inflation (in the case of TOF). Associated morbidity is common and overall survival poor. Management may be technically challenging and fistulae may recur. ${ }^{1,2}$

Despite surgical advances, local recurrence of oesophageal cancers occurs in up to a third of individuals. ${ }^{2}$ Dysphagia and (spontaneous) oesophago-respiratory fistulae are the most common presentations of local disease recurrence. Average survival associated with recurrence of oesophageal cancer is 3-8 months.

latrogenic fistulae most commonly result from long-term tracheal intubation, ${ }^{2}$ but have reported following local surgery and after percutaneous tracheostomisation. ${ }^{3}$ Fistulae have only rarely been reported following oesophageal stenting for malignancy, ${ }^{4}$ and we identified no reports of fistulae resulting from oesophageal dilatation.

Consequences of TOF and BOF range from impaired quality of life to the development of potentially life-threatening complications. Entry of saliva, liquid or food or into the pulmonary tree may precipitate bronchospasm, chest pain or recurrent respiratory tract infections. More acutely, aspiration pneumonia and pneumonitis may present as rapid onset of severe respiratory failure.

Clinical features may be determined by the anatomical site of the fistula, orifice size and the degree of material entering the pulmonary tree. The precipitous onset of respiratory failure in this case report suggests substantial airway soiling with food and perhaps blood via the large fistula.

Acute management entailed airway control, mechanical ventilation and haemodynamic support. The guidance for airway management in TOF is to position the ETT cuff at the carina to prevent soiling of the airway. However, because the oesophageal connection was at the level of the left main bronchus in this case report, airway isolation was not possible with the single lumen ETTs used. Purposeful endobronchial intubation of the right main bronchus confined any soiling to only the left lung before arrival in theatre.

In the management of BOF, use of a double- rather than single-lumen ETT would additionally protect the affected lung from further airway soiling via the trachea, but would offer no protection from ongoing soiling via the fistula. Placement of the ETT tip under fibreoptic visualisation might however prevent further trauma to the affected bronchus.

Positive pressure ventilation in the presence of BOF or TOF may result in pneumomediastinum or pneumothorax (as in this case) and it is therefore imperative to closely monitor for signs of these and to treat expeditiously.

In patients with recurrent malignancy, intervention usually takes the form of endoscopic stent placement, with surgical management of oesophageal fistulae reserved for the most difficult cases. Case series indicate that the parallel deployment of oesophageal and tracheal stents may be effective in the management of both local mass effects and TOF. ${ }^{5}$

Y-stents have found recognition in the palliation of symptoms arising from tracheobronchial tumours, and their use might be successfully adapted to the management of BOF. ${ }^{6}$ 


\section{REFERENCES}

1 Lerut T, Marulli. Early and late outcome after surgical treatment of acquired non-malignant tracheo-oesophageal fistulae DISCUSSION. European Journal of Cardio-Thoracic Surgery 2013; 43:

E160-E1

2 Blom RL, Lagarde SM, van Oudenaarde K, et al. Survival After Recurrent Esophageal Carcinoma Has Not Improved Over the Past 18 Years. Annals of Surgical Oncology 2013; 20: 2693-8

3 Drage SM, Soo CP, Dexter T. Delayed presentation of tracheo-oesophageal fistula following percutaneous dilatational tracheostomy. Anaesthesia 2002; 57: 932-3

4 Hamer PW, Griffiths EA, Devitt PG. Tracheo-oesophageal fistula after palliative treatment of oesophageal cancer. Journal of Gastrointestinal Oncology 2015; 6: E86-E8

5 Paganin F, Schouler L, Cuissard L, et al. Airway and Esophageal Stenting in Patients with Advanced Esophageal Cancer and Pulmonary Involvement. Plos One 2008; 3

6 Erb C, Pisani M, Puchalski J. The Utility of a Silicone Y-Stent in the Secondary Carina for a LifeAltering Bronchoesophageal Fistula. Chest 2013; 144 